\title{
WestVirginiaUniversity
}

THE RESEARCH REPOSITORY @ WVU

Graduate Theses, Dissertations, and Problem Reports

2018

\section{Effects of Serial- or Single-Alternative Training on Resurgence.}

Claudia C. Diaz-Salvat

Follow this and additional works at: https://researchrepository.wvu.edu/etd

\section{Recommended Citation}

Diaz-Salvat, Claudia C., "Effects of Serial- or Single-Alternative Training on Resurgence." (2018). Graduate Theses, Dissertations, and Problem Reports. 8190.

https://researchrepository.wvu.edu/etd/8190

This Thesis is protected by copyright and/or related rights. It has been brought to you by the The Research Repository @ WVU with permission from the rights-holder(s). You are free to use this Thesis in any way that is permitted by the copyright and related rights legislation that applies to your use. For other uses you must obtain permission from the rights-holder(s) directly, unless additional rights are indicated by a Creative Commons license in the record and/ or on the work itself. This Thesis has been accepted for inclusion in WVU Graduate Theses, Dissertations, and Problem Reports collection by an authorized administrator of The Research Repository @ WVU. For more information, please contact researchrepository@mail.wvu.edu. 


\title{
Effects of Serial- or Single-Alternative Training on Resurgence \\ Claudia C. Diaz-Salvat, B.S.
}

\author{
Thesis submitted to the \\ Eberly College of Arts and Sciences \\ at West Virginia University \\ in partial fulfillment of the requirements for the degree of \\ Master of Science in \\ Psychology \\ Claire C. St. Peter, Ph.D., BCBA-D, Chair \\ Barry Edelstein, Ph.D. \\ Kathryn M. Kestner, Ph.D., BCBA-D \\ Department of Psychology
}

Morgantown, West Virginia

2018

Keywords: differential reinforcement, problem behavior, resurgence, serial-response training

Copyright 2018 Claudia C. Diaz-Salvat 


\section{Abstract \\ Effects of Serial- or Single-Alternative Training on Resurgence}

Claudia C. Diaz-Salvat

Serial-response training involves the consecutive training of several alternative responses whereas single-response training involves the training of a single alternative response. Serialresponse training may mitigate resurgence of a target response when compared to teaching a single alternative response. However, previous evaluations did not control for the number of available response options across conditions. This study compared serial- and single-response training on resurgence of a target behavior when the number of response options was held constant. Serial-response training involved reinforcing each of four alternative responses sequentially. Single-response training involved reinforcing a single alternative response. Resurgence was tested by withholding all reinforcers and examining transient increases in response rates. No consistent differences across conditions occurred. Our results suggest that the number of available response options, and not serial-response training, was critical to outcomes. 


\section{Acknowledgements}

I would like to express my gratitude to my thesis chair and advisor, Dr. Claire St. Peter for her continuous support. Without her invaluable mentorship, this thesis would not have been possible. I would also like to thank Dr. Barry Edelstein and Dr. Kathryn Kestner for serving on the committee for this project and for providing me with helpful comments and suggestions. Finalmente, a mis padres, con mucho amor y cariño, les dedico mi esfuerzo. 


\section{Table of Contents}

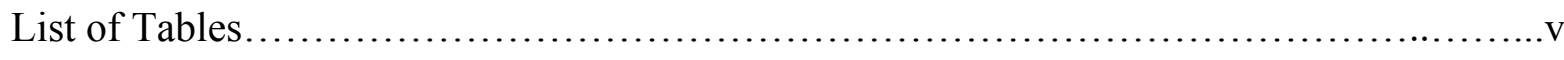

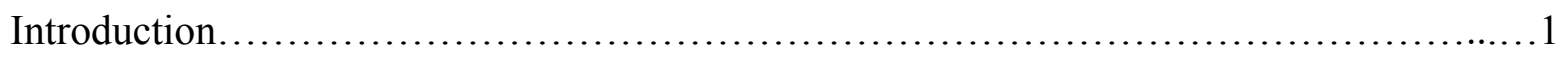

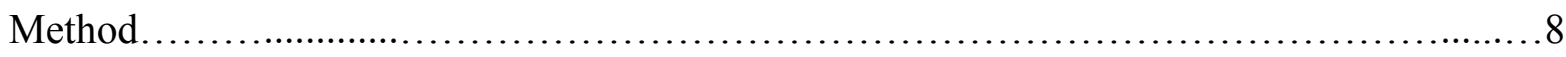

Results................................................................... 12

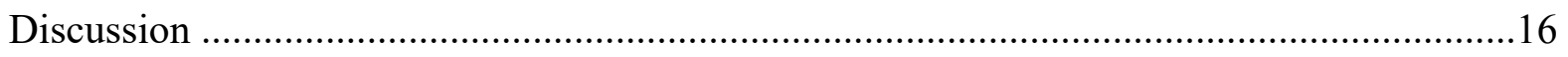

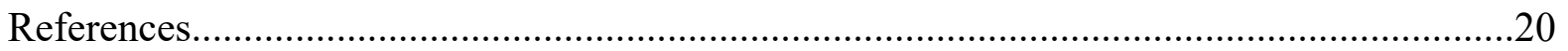

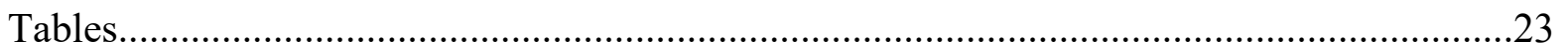

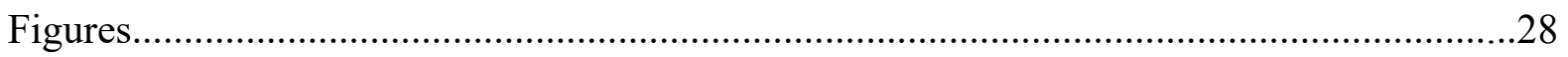




\section{List of Tables}

Tables

1. Background Colors and Response Colors For Each Component.......................23

2. RGB Values For Background Colors and Response Colors...............................24

3. Obtained Reinforcement Rates For the Serial- and Single-Training Components Across Exposures to the target-Reinforcement Phase...............................................25

4. Mean Alternative-Response Rates for the Serial- and Single-Training Components Across Exposures to the Extinction Phase.................................................................26

5. Obtained Reinforcement Rates For the Serial- and Single-Training Components Across Exposures to the Target-Reinforcement Phase...............................................27 


\section{Effects of Serial- or Single-Alternative Training on Resurgence}

Responses that had been previously reduced by behavioral treatment sometimes recur.

One type of such recurrence is resurgence. Resurgence is the transient recurrence of some dimension of previously established behavior when reinforcement conditions of current behavior are worsened (Lattal et al., 2017). Experimental evaluations of resurgence typically involve three phases. In the first phase, a target response is reinforced until stable rates of target responding occur. In the second phase, the target response is placed on extinction and an alternative response is reinforced. During this phase, target-response rates decrease and alternative-response rates increase. In the third phase, the alternative response is placed on extinction; neither target nor alternative is reinforced. During this phase, target-response rates temporarily increase relative to rates during the second phase. This increase in target responding is resurgence.

The three-phase resurgence procedure closely resembles the treatment of clinically relevant behavior using differential reinforcement of alternative behavior (DRA; Petscher, Rey, \& Bailey, 2009; St. Peter, 2015). The first two phases observed in a resurgence procedure mimic the arrangement generally seen when behavior analysts treat challenging behavior. In the first phase of a resurgence procedure, engaging in a target response (such as aggression) is reinforced. In clinical treatment, the challenging behavior has a history of reinforcement before treatment, and may be explicitly reinforced during a functional analysis (e.g., Iwata, Dorsey, Slifer, Bauman, \& Richman, 1982/1994). In the second phase, aggression is placed on extinction and an alternative behavior is reinforced using DRA. After DRA intervention, the initial challenging response may recur if reinforcement conditions for the alternative response worsen (Lattal et al., 2017), such as a reduction in reinforcement rate (Lieving \& Lattal, 2003; Marsteller \& St. Peter, 
2012; Volkert, Lerman, Call, \& Trosclair-Lasserre, 2009) or abrupt discontinuation of reinforcement (Lieving \& Lattal, 2003; Volkert et al., 2009).

Perhaps because of the parallels between common resurgence procedures and clinical behavioral treatments, recent work has focused on manipulating aspects of reinforcement-based treatments to reduce the likelihood of later resurgence. One such treatment manipulation involves the consecutive training of several alternative responses (termed serial-response training), which was recently evaluated by Lambert, Bloom, Samaha, and Dayton (2017). Lambert et al. (2017) taught topographically different requests that included American Sign Language (ASL), picture cards, microswitches, and hand raises to two children. The experimenters embedded a two-component multiple-schedule design within a two-tier nonconcurrent multiple baseline to compare two variations of DRA: teaching a single alternative and teaching three alternatives. Each variation was associated with a component of the multiple schedule; sessions associated with each variation were either conducted in different rooms or were associated with different stimuli to enhance discrimination between components.

During Phase 1, both components were identical except for the component-correlated stimuli (different colored t-shirts or colored paper); the experimenters reinforced each instance (i.e., a fixed-ratio [FR] 1 schedule) of the target response (tantrums or aggression) with the behavior's functional reinforcer (highly preferred toys or attention). Both participants engaged in moderate to high rates of target behavior and few appropriate requests.

During Phase 2, components continued to alternate, but target responding was no longer reinforced (i.e., extinction) in both components. However, the reinforcement procedures differed across components. During the single-training component, participants were taught to emit a single alternative response (e.g., hand raising or touching the therapist with a picture card 
containing the words "toys please") that resulted in 30-s reinforcer access. During the serialtraining component, participants were taught to emit one of three different alternative responses in sequential order. During this component, researchers initially reinforced one topography (e.g., ASL sign for "want") on an FR-1 schedule. Once the request occurred independently across five consecutive components and problem behavior was suppressed, the researchers discontinued reinforcement for the initial alternative request and taught a second alternative request (e.g., ASL sign for "play"). After the second topography consistently occurred while problem behavior remained suppressed, the researchers discontinued reinforcement for that alternative and taught a third alternative (e.g., activating a microswitch that said "may I have that?"). For both participants, target responding decreased and appropriate responding increased and stabilized throughout the phase.

During Phase 3, the researchers discontinued reinforcement for all responses (extinction) to evaluate differences in resurgence across the components previously associated with single training or serial training. Results were somewhat mixed. Both participants engaged in more appropriate requests during extinction following serial training than single training. These increased appropriate requests meant that, of all behavior emitted by a participant, target behavior accounted for a lower percentage following serial training than single training. However, one participant engaged in more total instances of target behavior during extinction following serial training than single training; opposite results were found for the other participant. Thus, the effects of serial training on resurgence of socially significant behavior remains unclear.

Although important, studying resurgence of socially significant challenging behavior can be difficult. One difficulty is controlling for reinforcement of challenging behavior between 
treatment sessions. For example, biting an adult to gain access to a toy may be placed on extinction during treatment sessions, but may be intermittently reinforced at home. Opportunities for extraneous reinforcement between sessions constitute confounding variables that may contribute to resurgence beyond the programmed independent variables, and may account for the discrepant results obtained by Lambert et al. (2017). An additional difficulty associated with studying resurgence of challenging behavior is that it may be unethical to deliberately cause relapse during resurgence phases. Although resurgence is a transient effect, challenging behaviors may occur at high rates or intensities (Volkert et al., 2009). This high-intensity behavior may put people at risk and may be clinically unacceptable.

Assessing resurgence of arbitrary (i.e., not socially significant) responses in controlled laboratory settings may avoid the difficulties described above. Such setups are often called human-operant arrangements, which are frequently characterized by selection of convenience samples, reinforcement of arbitrary responses, and manipulation of features of a resurgence procedure. A human-operant arrangement allows experimenters to evaluate effects of a treatment manipulation on subsequent resurgence without between-session reinforcement history as a confounding variable. Human-operant arrangements also allow experimenters to avoid exposing clients to periods of non-reinforcement that may evoke unacceptable rates or intensities of challenging behavior (Mace \& Critchfield, 2010).

Instead of reinforcing socially significant behaviors, Lambert, Bloom, Samaha, Dayton, and Rodewald (2015) conducted a laboratory-based evaluation of whether serial-response training affected resurgence. Lambert et al. taught five topographically different movements with various devices (toggle switch, rocker switch, slide switch, cord switch, and button) to individuals with developmental disabilities. The experimenters used a within-subject, two- 
component multiple schedule to compare serial- and single-response training. Each training type was associated with a component of the multiple schedule; sessions associated with each variation were conducted in different rooms to enhance discrimination between components.

During Phase 1, both components were identical except for the location; the experimenters reinforced the same target response (e.g., toggle-switch closure) on an FR-1 schedule with a small piece of preferred food. Only the target response was available to the participant across both components during this phase. During Phase 2, components continued to alternate, but target responding was placed on extinction in both components. However, the reinforcement procedures differed across components. During the single-training component, participants were taught to emit a single alternative response (e.g., rocker-switch closure); responding on this device resulted in preferred food on an FR-1 schedule throughout the phase. Only the devices for the target and single alternative response were available to the participant during this component. During the serial-training component, participants were taught to emit one of three different alternative responses in sequential order. During this component, researchers initially reinforced responding on one alternative device (e.g., slide switch) on an FR-1 schedule during a training period and five subsequent sessions. Then, the researchers discontinued reinforcement for the initial alternative device, introduced a second alternative device (e.g., cord switch), and reinforced responding on the second device on an FR-1 schedule for a training period and five sessions. During this portion of the phase, the target device and both alternative devices were available to the participant. After the five sessions of reinforcement for the second alternative, the researchers discontinued reinforcement for the second alternative and introduced a third alternative (e.g., button), which was reinforced on an FR-1 schedule for a 
training period and five subsequent sessions. By the end of the phase, the target response and all three alternative responses were available to the participant in the serial-training component. During Phase 3, the researchers discontinued reinforcement for all responses (extinction) to evaluate differences in resurgence across the components previously associated with single training or serial training. Resurgence of target responses occurred to a greater extent in the component associated with single training than in the component associated with serial training, suggesting that teaching multiple alternative responses during DRA reduced subsequent resurgence. However, the experimenters continued to have different numbers of devices available across the components (two devices in the single-training component and four devices in the serial-training component). It is possible that the difference in the number of available responses, rather than the history of serial training, impacted resurgence.

Carrasquillo and Ringdahl (in press) attempted to replicate the treatment effects described by Lambert et al. (2015). The experimenters conducted a laboratory-based evaluation of whether serial training affected resurgence using a computer program to simulate the three-phase resurgence procedure. They taught five topographically identical responses, single mouse clicks to one of six colored squares, to undergraduate students. Similar to Lambert et al. (2015), the experimenters used a within-subject, two-component multiple schedule to compare serial training and single training, but participants were undergraduate students rather than individuals with disabilities. A 2-min serial-training component and a 2-min single-training component alternated across the three typical resurgence phases. During Phase 1, both components were identical except for a distinct background color; the experimenters reinforced the same target response on a variable interval (VI) 10-s schedule with a Windows ${ }^{\circledR}$ "chimes" tone and the addition of a point to their "Session Credits." During Phase 2, the serial- and single-training 
components continued to alternate, but target responding was on extinction during both components. The reinforcement procedures differed across components. During the singletraining component, clicks to the brown square resulted in a delivery of a point on a (VI) 10-s schedule throughout the phase. Only the squares for the target and single alternative response were available during this component. During the serial-training component, the experimenters reinforced one of four different alternative responses in sequential order. The target response option and four alternative response options were available. During this component, the experimenters reinforced responding on each colored square on a VI 10-s schedule.

Experimenters reinforced responding in a sequential order such that responding on the first square was reinforced during the first $30 \mathrm{~s}$ of the component presentation, responding on the second square was reinforced during the second $30 \mathrm{~s}$, and so on until the end of the 2 min component presentation.

During Phase 3, all responses were placed on extinction to evaluate differences in resurgence following serial- and single-response training. Like Lambert et al. (2015), resurgence of target responses occurred to a greater extent in the component associated with single training relative to the component associated with serial training, suggesting that teaching multiple alternative responses during DRA may reduce subsequent resurgence. However, similar to Lambert et al. (2015), the researchers continued to have different numbers of response options across the components (two response options in the single-training component and five response options in the serial-training component). Again, it is possible that the differences in the number of available response options, rather than the history of serial training, impacted resurgence. Equating the number of available response options across single- and serial-training components would better isolate effects of serial training. The purpose of the current study was to compare 
resurgence following single- and serial-response training, while keeping the number of available response options constant across components.

\section{Method}

\section{Participants and Setting}

Seven female and two male undergraduate students enrolled in a psychology course at West Virginia University participated. Participants were 18-21 years old (mean, 19.1 years) and were Caucasian. One 20-year-old participant did not complete the second 60-min session due to a program malfunction; data for this participant were excluded from analysis. Three participants completed interviews (P6-P8); none reported that they had a color-vision deficiency or currently took any psychotropic medication. Each participant signed an informed consent prior to participation.

We recruited participants by creating appointments through an online researchscheduling system (SONA) provided by the university. Participants received course credit through the SONA system for the time they spent participating, independently of their responding during the experiment. The amount of credit earned for every 30 min of participation varied by course, at the course instructors' discretions.

We conducted the experiment in a $4.1-\mathrm{m}$ by $3-\mathrm{m}$ university laboratory room that contained two tables, a computer desk, computer, and desk chair. Participants completed the experiment in individual, 140-min appointments, which were broken into two 60-min sessions separated by a 20-min break. Data sets that did not show clear changes in responding between Phases 1 and 2 would have been excluded, but this never occurred.

\section{Apparatus and Experimental Stimuli}


We used custom-created Visual Basic program running on a Dell Optiplex 755 desktop computer with a $48 \mathrm{~cm}$ monitor for all experimental procedures. Across all components and phases, six $24 \mathrm{~mm}$ circles of differing colors moved across the screen at a speed of $25 \mathrm{~mm} / \mathrm{s}$, and a cumulative point counter appeared at the bottom left of the screen. Each time the participant met the criterion specified by the reinforcement schedule, the cumulative point counter incremented by a point and briefly flashed orange.

We used a multiple schedule embedded in a reversal design to demonstrate experimental control. Each participant experienced two replications of three 20-min phases. Within each phase, participants experienced two components. Components were signaled by the background color on the computer screen. Component A was associated with a vermillion background and multiple alternative responses that were reinforced during Phase 2 (serial training). Component B was associated with a blue background and reinforcement of a single alternative response during Phase 2. Table 1 summarizes the background color and response colors for each component. Table 2 summarizes the RGB values for the background and response colors, which were selected because they should be distinct despite colorblindness (see Wong, 2011). Each component presentation was $2 \mathrm{~min}$, and components strictly alternated five times in each phase. The component presented first was counterbalanced across participants and phases.

\section{Procedure}

At the beginning of an appointment, the experimenter gave the participant a copy of the informed-consent document and said:

This is an experiment about how people learn to respond. If you agree to participate, you will be seated in front of a computer and will use only the mouse to earn as many points as possible during the session. Rather than have you sit there for two hours straight, you 
will be able to take a break midway through. Please remain seated at the computer until the thank you message appears on the screen. Do you have any questions?

After answering any questions, the experimenter reviewed the consent form with the participant. When consent was obtained, the experimenter asked the participant to leave all electronics on the table away from the computer to prevent overt timing of the phases or reinforcement schedules and escorted the participant to the computer.

Once the participant was seated in front of the computer, the experimenter stated: This is where you will be working. Remember to use only the mouse to earn as many points as possible. The instructions you see on the screen ["Press OK to start"] are the only ones that you will get: it is up to you to figure out how to earn points. The bottom left of the screen labeled "Score" will display your total points throughout the program. The session will be about $60 \mathrm{~min}$. At the end of that time, a thank you message will appear on the screen. When it appears, please come out and get me. You can then take a break while I reset the computer. You will then do one more 60-min session before finishing the experiment. Good luck!

After the first 60-min session, the experimenter asked the participant to take a break. The experimenter pointed the participant towards the restrooms, the water fountains, and the vending machines. The experimenter also explained that resetting the computers would take 20 min and that the hallway door would be closed until the computer was reset.

Following the break, the experimenter escorted the participant back to the computer and stated, "This will be similar to the last session. You will complete the experiment in about 60 min." Upon completion of the experiment, the experimenter thanked the participant and offered a copy of the consent. The experimenter asked the participant to report age, gender, ethnicity, and 
the course for which the participant wished to earn extra credit. Participants 6, 7, and 8 also completed an interview in which the experimenter asked if the participant had a color vision deficiency, took any psychotropic medication, and what they thought they had to do to earn points. The experimenter then debriefed the participant on the purpose of the study and answered any questions.

\section{Conditions.}

Target reinforcement. During the target-reinforcement phase, the two alternating components were identical except for the background color of the computer screen. Points were delivered for the first click on the black circle after $2 \mathrm{~s}$ elapsed (a fixed interval [FI] 2-s schedule). Five alternative responses (orange, sky blue, blue green, yellow, reddish purple) were concurrently available, but clicks on these circles were not reinforced.

Alternative reinforcement. During the alternative-reinforcement phase, points were no longer delivered for clicking the black circle (extinction). Points were delivered for clicking a designated alternative response on an FI 2-s schedule. During the serial-training component, four alternative responses (orange, sky blue, blue green, yellow) were reinforced sequentially on independent FI 2-s schedules for $30 \mathrm{~s}$ of each component presentation. Clicking the orange circle was reinforced for the first $30 \mathrm{~s}$, clicking the sky-blue circle was reinforced for the second $30 \mathrm{~s}$, clicking the blue-green circle was reinforced for the third 30s, and clicking the yellow circle was reinforced for the fourth $30 \mathrm{~s}$ of the 2 -min component. The four alternative responses were reinforced in the same order (orange, sky blue, blue green, yellow) during each presentation of the serial-training component. During the single-training component, clicking on the reddishpurple circle was reinforced on a FI 2-s schedule throughout the 2-min component. 
Resurgence. During the resurgence phase, the two alternating components were identical except for the background color of the computer screen. All responses were placed on extinction (no points were delivered) during both components. The target response and the five alternative responses were concurrently available during both components for the duration of the resurgence phase. Following the last component of the session, a thank you message ["Session Complete. Thank You.’] appeared.

\section{Response Measurement}

The computer program recorded data about location and time of individual clicks. The program also recorded the time of each point delivery and the contingency resulting in the delivery. Upon the completion of the study, a data file generated by the program was used to identify responses per min during each component presentation.

\section{Data Analysis}

We analyzed data using visual inspection of graphs. Although we analyzed response rates for each alternative-response option, we did not identify differential response patterns across the five alternatives during extinction. Therefore, we aggregated alternative responding during each component for all analyses reported. Analyses by individual alternative response are available from the author.

\section{Results}

Figures 1 and 2 display target-response rates from the target reinforcement, alternative reinforcement, and resurgence phases for each participant. Figure 1 displays data for the five participants that were not interviewed; Figure 2 displays data for the three participants that were interviewed. Filled circles depict target responding during the single-training component and open circles depict target responding during the serial-training component. For all participants, 
response rates changed predictably as the reinforcement schedules changed across phases.

During the target-reinforcement phase, target responding occurred at high rates in both components for all participants. During DRA, target responding suppressed to near-zero rates in both components for all participants. During the resurgence phase, target-response rates consistently increased at the start of the resurgence phase for some participants (P1, P2, P3, P6) relative to rates of target responding at the end of the alternative-reinforcement phase. Targetresponse rates varied for some participants $(\mathrm{P} 4, \mathrm{P} 5, \mathrm{P} 7, \mathrm{P} 8)$ across replications. This variation may have been due to responding resurging to a greater extent during the first replication relative to the second. These results demonstrated that points functioned as a reinforcer.

Figures 1 and 2 allow us to compare target responding during the extinction phase to target responding in previous phases. However, it is difficult to compare target responding in the serial-training component to target responding in the single-training component during the resurgence phases due to the range of the y-axes. Figures 3 and 4 display target-response rates across component presentations during both extinction phases. Target-response rates across serial- and single-training components were undifferentiated during both exposures to extinction for Participants 2, 3, 6, 7, and 8. Target-response rates across component presentations were differentiated during both exposures to extinction for Participants 4 and 5. Participant 4 engaged in more target responses per minute during the single-training component, while Participant 5 engaged in more target responses per minute during the serial-training component suggesting a lack of between-subject replication. Target-response rates were differentiated during the second exposure to extinction for Participant 1, but were not differentiated during the first exposure. Participant 1 engaged in more responses per minute during the single- relative to the serialtraining component. Thus, there were no consistent differences across training type. 
Although Figures 2 and 3 allowed us to compare session by session responding across the training types, they are not as useful for comparing total number of responses across components. Overall responses may be of interest when the target response is a socially significant dangerous behavior. When target responses are dangerous and may put individuals at risk, effective treatments should result in a smaller total number of responses relative to other treatment options. Therefore, we calculated total target responses during the serial- and singletraining component during extinction. Figures 5 and 6 display total target responses across exposures to extinction for all participants. Within-subject replication occurred for only two of eight participants (P4 and P5) in Figure 5. Participant 4 engaged in more total target responses during the single-training component, participant 5 engaged in more total responses during the serial-training component during both exposures to extinction, suggesting a lack of betweensubject replication. Reductions in the amount of responding during the second exposure to extinction occurred for Participants 3, 4, 5, 6, 7, and 8. Reductions in the number of responses during the second exposure to extinction did not occur for Participants 1 and 2 . These effects support previous research that suggests that repeated exposures to extinction decrease resurgence relative to initial exposures to extinction (Diaz-Salvat, Kestner, \& St. Peter, 2018; Sweeney \& Shahan, 2013).

Previous research has shown that resurgence occurs to a greater extent when targetreinforcement rates are high rather than low (Cançado, Abreu-Rodrigues, \& Aló, 2015; Podlesnik \& Shahan, 2009, Experiment 2). When comparing the effect of two treatments on subsequent resurgence, it is important to compare target-reinforcement rates and reinforcement ratios to rule out the possibility that differences between treatments may have been due to differences in reinforcement rates instead of the actual treatment manipulations. Table 3 includes 
target-reinforcement rates and reinforcement ratios for both exposures to the serial- and singletraining components. There were no systematic differences across participants when comparing reinforcement rates and reinforcement ratios.

Resurgence of target behavior did not seem to be influenced by serial training, and differences in reinforcement rates could not account for this failure to replicate previous findings. However, it remained possible that serial training impacted the extent to which alternative responding persisted during extinction. Such an outcome might be desirable despite lack of difference in resurgence. Therefore, we compared rates of alternative responding across each phase and exposure to the experimental arrangement. Figures 7 and 8 display alternativeresponse rates from the target reinforcement, alternative reinforcement, and resurgence phases for all participants. Few alternative responses occurred during the target-reinforcement phase, but alternative responding occurred at high rates when those responses resulted in reinforcement (i.e., during DRA phase). Rates of alternative responding declined during the extinction phase, when those responses no longer resulted in reinforcement. These results further demonstrated that points functioned as a reinforcer.

To facilitate comparisons between alternative-response rates across the serial- and singletraining components during exposures to extinction, we calculated mean response rates across components and replications. Table 4 includes mean alternative response rates across serial- and single-training components for each participant during both exposures to extinction. Participant 1 engaged in more alternative-responses per minute during the serial- relative to the single-training component. Participants 2, 3, 5, and 8 engaged in more alternative responses per minute during the first exposure to the serial-training component, but engaged in more alternative responses per minute during the second exposure to the single-training component. Participants 4 and 7 
engaged in more alternative responses per minute during the first exposure to the single-training component, but engaged in more alternative responses per minute during the second exposure to the serial training component. Participant 6 engaged in an equal amount of alternative responses per minute across the first exposure to the serial- and single-training components, but engaged in more alternative responses per minute during the second exposure to the single-training component.

Similar to high target-reinforcement rates, treatments with high alternative-reinforcement rates result in more durable effects (i.e. more persistence and less resurgence; Craig \& Shahan, 2016). Table 5 includes alternative-reinforcement rates and alternative reinforcement ratios for both exposures to the serial- and single-training components. There were no systematic differences within or across participants when comparing alternative-reinforcement rates and alternative reinforcement ratios.

\section{Discussion}

Our study addressed a potential confound in previous studies (Carrasquillo \& Ringdahl, in press; Lambert et al., 2015) that compared effects of serial and single-response training on subsequent resurgence without controlling for the number of available response options. These studies had 4 (Lambert et al., 2015) or 5 (Carrasquillo \& Ringdahl, in press) available response options during resurgence tests following serial-response training and only 2 response options following single-response training. Although these studies showed mitigated resurgence following serial training relative to single-response training, the obtained reductions could be due to either the training history or the number of available responses. We controlled for the number of available response options by having six response options available during both the serial and single-response training components, and obtained no consistent differences in resurgence. Our 
results suggest that mitigated resurgence following serial training relative to single-response training obtained in previous studies (Carrasquillo \& Ringdahl, in press; Lambert et al., 2015) may not have been due to serial training per se.

Although our results suggest that the number of available responses is critical to outcomes, we did not conduct further manipulations to isolate the number of available response options as the cause of decreased resurgence. We are currently conducting additional studies in which we manipulate the number of available response options while holding the number of reinforced responses constant. We hope that these studies will further demonstrate that the number of response options was indeed the critical variable impacting resurgence in previous findings (e.g., Carrasquillo \& Ringdahl, in press; Lambert et al., 2015).

Because our experimental procedures differed from those in previous evaluations, it is also possible (although seemingly unlikely) that our failure to replicate was due to procedural variations. Similar to Carrasquillo and Ringdahl (in press), we used a computer program to simulate the three-phase resurgence procedure. However, our response options (colored circles) moved across the screen, while the squares used by Carrasquillo and Ringdahl (in press) were stationary. Responding during the extinction phases may have been affected by our moving response options, although there is no evidence to suggest that this is the case. Additionally, all of our responses were topographically identical. It is unclear whether these were actually distinct responses in the same way that Lambert et al. (2015) taught topographically distinct responses (different movements with various devices). The use of topographically distinct alternatives may increase potential positive effects of serial training (although this is unlikely in light of reduced resurgence in the procedures used by Carrasquillo and Ringdahl (in press) when participants were taught topographically identical responses). Instead, our results may be indicative of 
induction, the spread of effects of reinforcement outside of the responses that were reinforced, instead of resurgence per se (Lattal \& St. Peter Pipkin, 2009). It is possible that the effects of reinforcement spread to response options that were previously not reinforced. Induction may result in differential amounts of resurgence when responses are topographically identical.

Our experimental arrangement had some notable differences to arrangements involving socially significant behaviors. First, we did not have to complete DRA training. If we had to teach and train, like teaching a child one or more alternative responses that are not part of their repertoire, we may have gotten different results. However, this seems unlikely, given that Carrasquillo and Ringdahl (in press), which used procedures similar to ours, obtained similar results to Lambert et al. (2015), which included a training phase. Second, our topographically identical responses required equal effort across target and alternative responses. Socially significant alternative responses, like communicative responses, may be more effortful than the target response. Additionally, socially significant alternative responses may have historically had a longer delay to reinforcement relative to the challenging response. Third, our participants experienced an extinction phase that was the same duration as the target and DRA phase. In clinical settings, individuals may not experience extended periods of extinction. Fourth, we did not reinforce all alternative responses on a concurrent schedule prior to the start of Phase 3 . Such a concurrent reinforcement history would likely occur in any clinical application. Subsequent studies should investigate effects of concurrent reinforcement histories for multiple alternative topographies on resurgence.

We demonstrated experimental control with a multielement design where we alternated between two components (serial- and single-response training) with different background colors. Our multielement design is a potential limitation; participants might not have experienced two 
distinct treatments. Instead, participants may have experienced this as training five alternative responses in a short period of time rather than distinct single and serial training. Future researchers should consider using a group design in which participants are only exposed to one treatment during the second phase. A group design would ensure that participants experience serial- or single-response training.

We included within-subject reversals in an attempt to evaluate whether effects in the first exposure were replicable within subjects. Some participants engaged in more responding during the first component in at least one extinction presentation relative to subsequent presentations. This responding across participants may have impacted our effects and our ability to draw conclusions from our findings. Although previous studies that compared serial- to singleresponse training (Carrasquillo \& Ringdahl, in press; Lambert et al., 2015) did not include within-subject reversals, resurgence evaluations that have included within-subject reversals have identified a decreased resurgence effect during the second exposure to extinction relative to the first exposure (Romano \& St. Peter, 2016). Our results aligned with those of previous studies suggesting that additional exposures to extinction may mitigate the resurgence effect. Additionally, responding during the extinction phase did not fully extinguish for all participants suggesting that a longer exposure to extinction may have been necessary for participants to stop responding. 


\section{References}

Cançado, C. R. X., Abreu-Rodrigues, J., \& Aló, R. M. (2015). Reinforcement rate and resurgence: A parametric analysis. Mexican Journal of Behavior Analysis, 41, 84-115. doi: 10.5514/rmac.v41.i2.63739

Carrasquillo, T., \& Ringdahl, J. E. (in press). The effect of teaching multiple response alternatives on response resurgence and persistence. Journal of Applied Behavior Analysis.

Craig, A. R., \& Shahan, T. A. (2016). Behavioral momentum theory fails to account for reinforcement rate on resurgence. Journal of the Experimental Analysis of behavior, 105, 375-392. doi: 10.1002/jeab.207

Iwata, B. A., Dorsey, M. F., Slifer, K. J., Bauman, K. E., \& Richman, G. R. (1994). Toward a functional analysis of self-injury. Journal of Applied Behavior Analysis, 27, 197-209. doi: 10.1901/jaba.1994.27-197

Lambert, J. M., Bloom, S. E., Samaha, A. L., \& Dayton, E. (2017). Serial functional communication training: Extending serial DRA to mands and problem behavior. Behavioral Interventions, 32, 311-325. doi: 10.1002/bin.1493

Lambert, J. M., Bloom, S. E., Samaha, A. L., Dayton, E., \& Rodewald, A. M. (2015). Serial alternative response training as intervention for target response resurgence. Journal of Applied Behavior Analysis, 48, 765-780. doi: 10.10002/jaba.253

Lattal, K. A., Cançado, C. R. X., Cook, J. E., Kincaid, S. L., Nighbor, T. D., \& Oliver, A. C. (2017). On defining resurgence. Behavioral Processes, 141, 85-91. Doi: 10.1016/j.beproc. 2017.04 .018 
Lieving, G., \& Lattal, K. A. (2003). Recency, repeatability, and reinforce retrenchment: an experimental analysis of resurgence. Journal of the Experimental Analysis of Behavior, 80, 217-233. doi: 10.1901/jeab.2003.80-217

Mace, C. F., \& Critchfield, T. S. (2010). Translational research in behavior analysis: Historical traditions and imperative for the future. Journal of the Experimental Analysis of Behavior, 93, 293-312. doi: 10.1901/jeab.2010.93-293

Marsteller, T. M, \& St. Peter, C. C. (2012). Resurgence during treatment challenges. Mexican Journal of Behavior Analysis, 38, 7-23.

Petscher, E. S., Rey, C., \& Bailey, J. S. (2009). A review of empirical support for differential reinforcement of alternative behavior. Research in Developmental Disabilities, 30, 409425. doi:10.1016/j.ridd.2008.08.008

Podlesnik, C. A., \& Shahan, T. A. (2009). Behavioral momentum and relapse of extinguished operant responding. Learning and Behavior, 37, 357-364. doi: 10.3758/LB.37.4.357

Romano, L. M., \& St. Peter, C. C. (2016). Omission training results in more resurgence than alternative reinforcement. The Psychological Record, 67, 315-324. doi: 10.1007/s40732016-0214-z

St. Peter, C. C. (2015). Six reasons why applied behavior analysts should know about resurgence. Mexican Journal of Behavior Analysis, 41, 252-268.

Sweeney, M. M., \& Shahan, T. A. (2013). Behavioral momentum and resurgence: Effects of time in extinction and repeated resurgence tests. Learning and Behavior, 41, 414-424. doi: 10.3758/s13420-013-0116-8 
Volkert, V. M., Lerman, D. C., Call, N. A., Trosclair-Lasserre, N. (2009). An Evaluation of Resurgence During Treatment with Functional Communication Training. Journal of Applied Behavior Analysis, 42, 145-160. doi:10.1901/jaba.2009.42-145

Wong, B. (2011). Points of View: Color blindness. Nature Methods, 8, 441. doi:10.1038/nmeth.1618 
Table 1

Background Color and Response Colors For Each Component.

\begin{tabular}{ccccc}
\hline \multirow{2}{*}{ Component } & \multicolumn{4}{c}{ Stimuli } \\
\cline { 2 - 5 } & Background Color & $\begin{array}{c}\text { Target } \\
\text { Response }\end{array}$ & $\begin{array}{c}\text { Reinforced } \\
\text { Alternative(s) }\end{array}$ & $\begin{array}{c}\text { Unreinforced } \\
\text { Alternative(s) }\end{array}$ \\
\hline A & Black & $\begin{array}{c}\text { Orange, } \\
\text { Sky blue, } \\
\text { Bluish green, } \\
\text { Blue }\end{array}$ & Yellow & Reddish \\
purple \\
B
\end{tabular}


Table 2

RGB Values For Background Colors and Response Colors.

\begin{tabular}{ccl}
\hline Color & RGB Value & Use \\
\hline Vermillion & $(213,94,0)$ & Background A \\
Blue & $(0,114,178)$ & Background B \\
Black & $(0,0,0)$ & Target A + B \\
Orange & $(230,159,0)$ & Alternative A \\
Sky Blue & $(86,180,233)$ & Alternative A \\
Bluish Green & $(0,158,115)$ & Alternative A \\
Yellow & $(240,228,66)$ & Alternative A \\
Reddish Purple & $(204,121,167)$ & Alternative B \\
\hline
\end{tabular}


Table 3

Obtained reinforcement rates for the serial-and single-training components across exposures to the target reinforcement phase.

\begin{tabular}{lllllll}
\hline & \multicolumn{5}{c}{ Target Reinforcement Phase } \\
\cline { 2 - 7 } Participants & \multicolumn{3}{c}{ First Exposure } & \multicolumn{3}{c}{ Second Exposure } \\
\cline { 2 - 7 } & Serial & Single & Ratio & Serial & Single & Ratio \\
\hline 1 & 23.5 & 17.3 & 0.74 & 27.8 & 27.7 & 0.94 \\
2 & 23.7 & 24.6 & 1.04 & 27.3 & 20.1 & 0.86 \\
3 & 26.6 & 25 & 0.94 & 25.5 & 19.4 & 1.04 \\
4 & 28.7 & 28.8 & 1.00 & 29.1 & 28.8 & 0.91 \\
5 & 26.6 & 27 & 1.02 & 28.2 & 27.2 & 0.94 \\
6 & 26.9 & 28.9 & 1.07 & 25 & 28.8 & 0.94 \\
7 & 27.9 & 26.7 & 0.96 & 29.4 & 27.8 & 0.88 \\
8 & 27.9 & 28.5 & 1.02 & 27.4 & 28.3 & 0.86 \\
\hline
\end{tabular}


Table 4

Mean alternative response rates for the serial- and single-training components across exposures to the extinction phase.

\begin{tabular}{lllllll}
\hline & \multicolumn{4}{c}{ Mean Alternative Responses During Extinction } \\
\cline { 2 - 7 } Participants & \multicolumn{3}{c}{ First Exposure } & \multicolumn{3}{c}{ Second Exposure } \\
\cline { 2 - 7 } 1 & Serial & Single & Ratio & Serial & Single & Ratio \\
\hline 2 & 27.6 & 19.7 & 1.4 & 49.2 & 35 & 1.41 \\
3 & 18 & 13.7 & 1.31 & 19.3 & 20.1 & 0.96 \\
4 & 14.1 & 12.1 & 1.17 & 8.9 & 10.1 & 0.88 \\
5 & 16.5 & 28 & 0.59 & 3.3 & 2.9 & 1.14 \\
6 & 15.2 & 7.8 & 1.95 & 3.6 & 4.1 & 0.88 \\
7 & 35.8 & 35.4 & 1 & 20 & 27.8 & 0.72 \\
8 & 31 & 34.8 & 0.89 & 18.7 & 18.1 & 1.03 \\
\hline
\end{tabular}


Table 5

Obtained reinforcement rates for the serial-and single-training components across exposures to the alternative reinforcement phase.

\begin{tabular}{lllllll}
\hline & \multicolumn{5}{c}{ Alternative Reinforcement Phase } \\
\cline { 2 - 7 } Participants & \multicolumn{4}{c}{ First Exposure } & \multicolumn{3}{c}{ Second Exposure } \\
\cline { 2 - 7 } & Serial & Single & Ratio & Serial & Single & Ratio \\
\hline 1 & 25.9 & 27.7 & 0.94 & 22.9 & 27.5 & 0.83 \\
2 & 17.2 & 20.1 & 0.86 & 23.8 & 26 & 0.92 \\
3 & 20.2 & 19.4 & 1.04 & 24.3 & 24.8 & 0.98 \\
4 & 26.3 & 28.8 & 0.91 & 27.8 & 29.7 & 0.94 \\
5 & 25.7 & 27.2 & 0.94 & 27.2 & 29.2 & 0.93 \\
6 & 27.1 & 28.8 & 0.94 & 25.7 & 26.9 & 0.96 \\
7 & 24.6 & 27.8 & 0.88 & 23 & 28.4 & 0.81 \\
8 & 24.3 & 28.3 & 0.86 & 25.3 & 28.4 & 0.89 \\
\hline
\end{tabular}




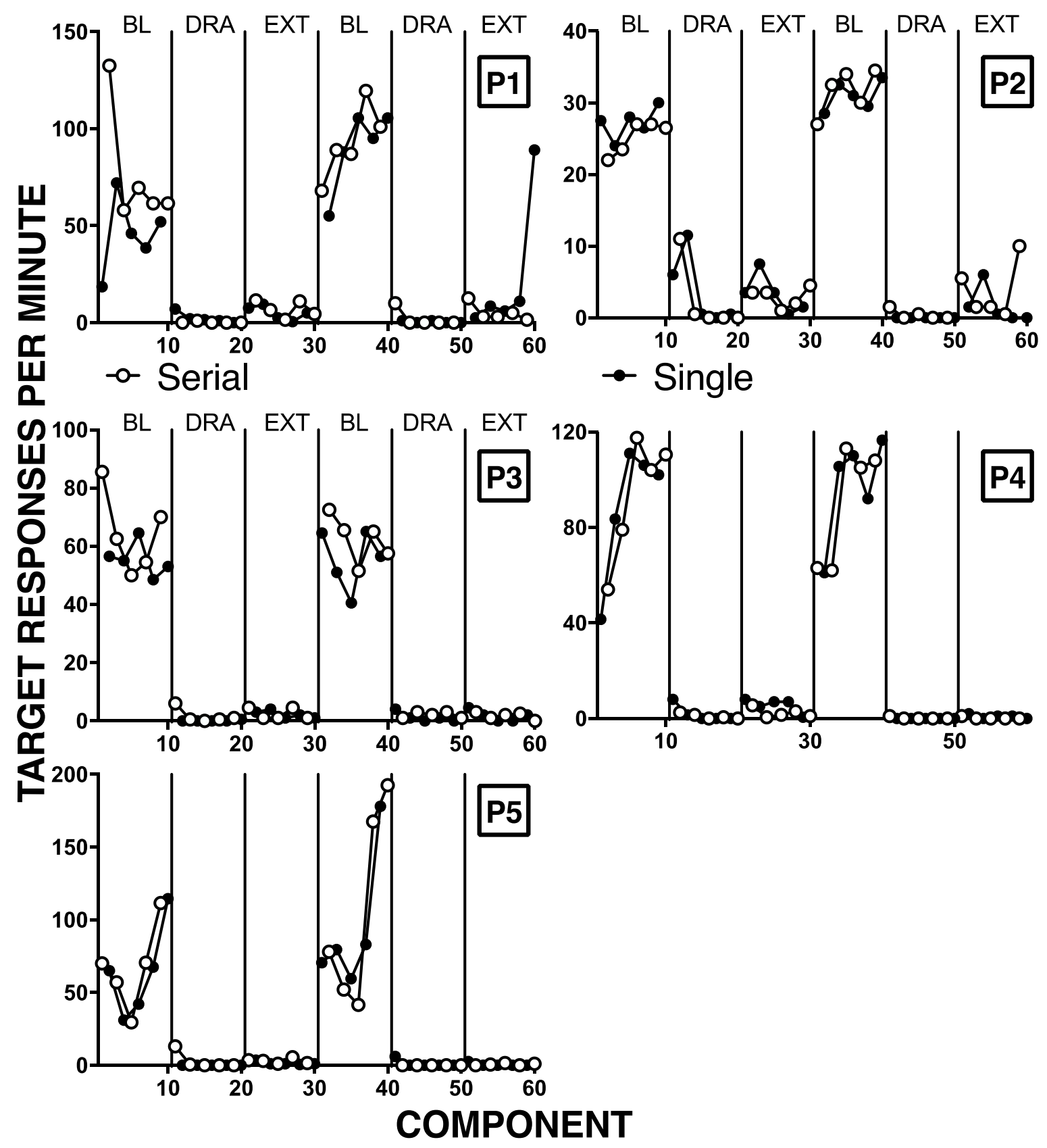

Figure 1. Results for participants who were not interviewed. Each graph shows results for a participant. Filled circles denote target-response rates during the single-training component, and open circles denote target-response rates during the serial-training component. Y-axes values vary across participants. Condition labels show baseline (BL), DRA, and extinction phases (EXT). 


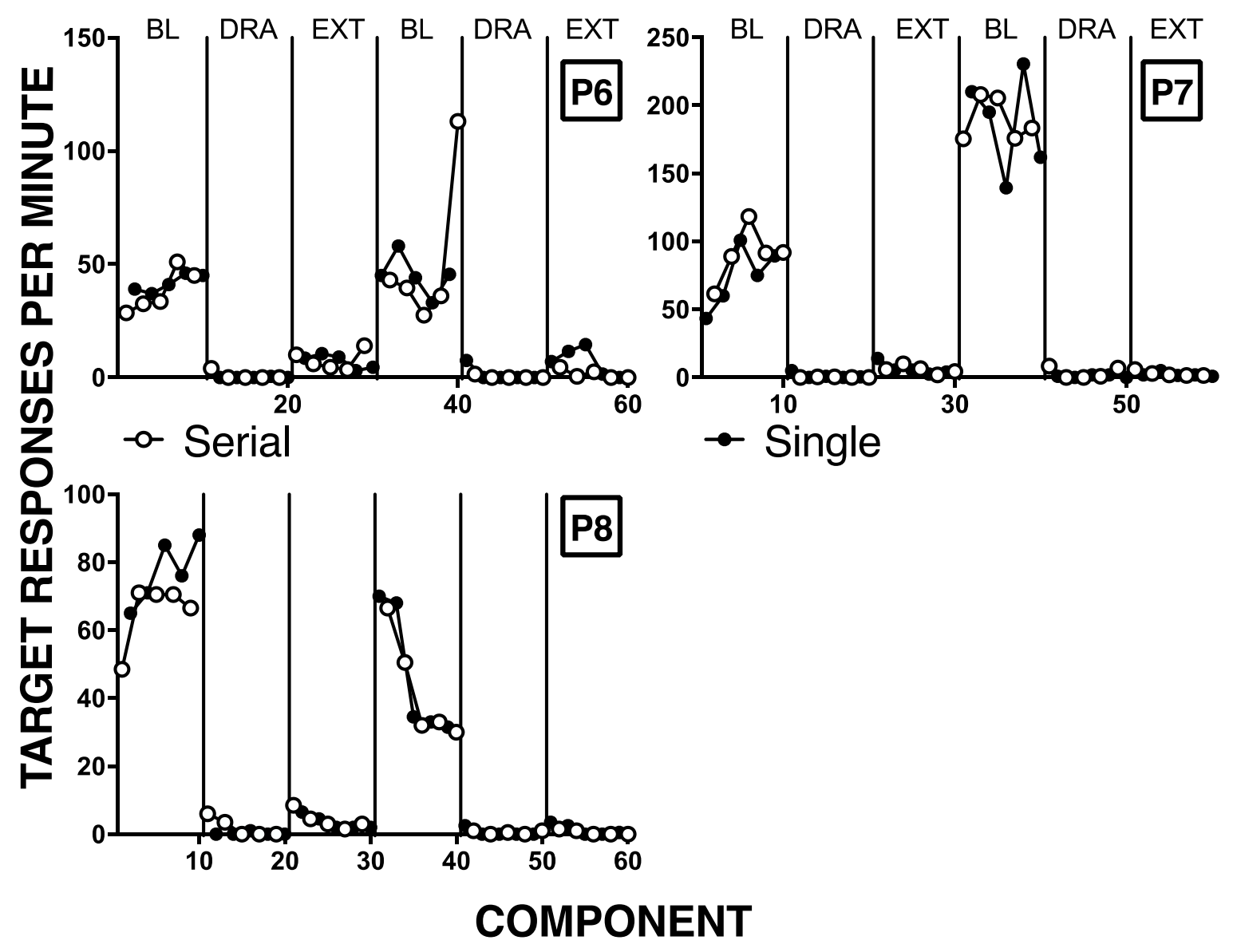

Figure 2. Results for participants who were interviewed. Each graph shows results for a participant. Filled circles denote target response rates during the single-training component, and open circles denote target-response rates during the serial-training component. Y-axes values vary across participants. Condition labels show baseline (BL), DRA, and extinction phases (EXT). 


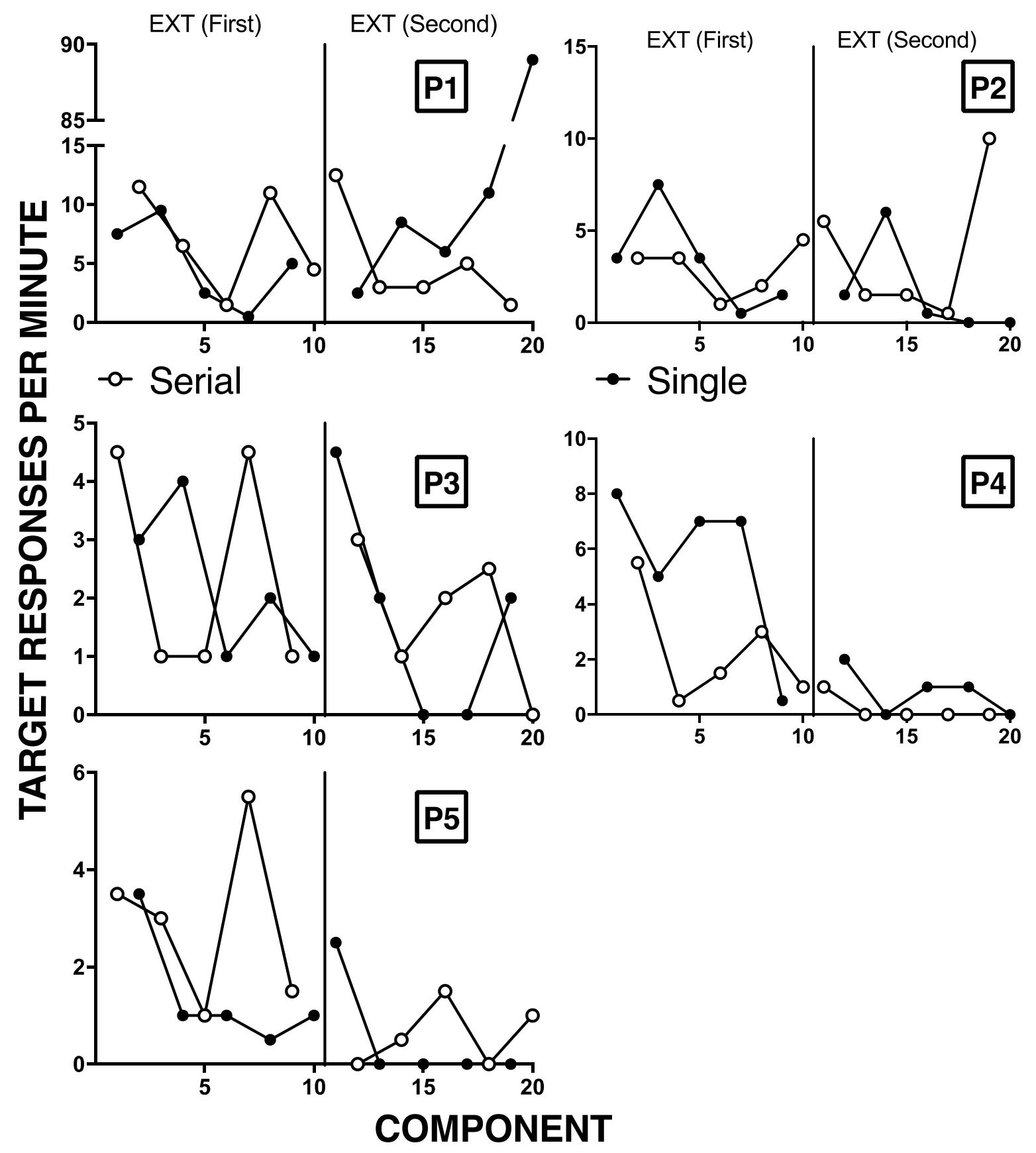

Figure 3. Results for participants who were not interviewed. Each graph shows results for a participant. Filled circles denote target-response rates during the single-training component, and open circles denote target-response rates during the serial-training component. Y-axes values vary across participants. Condition labels show the first and second extinction phase. 


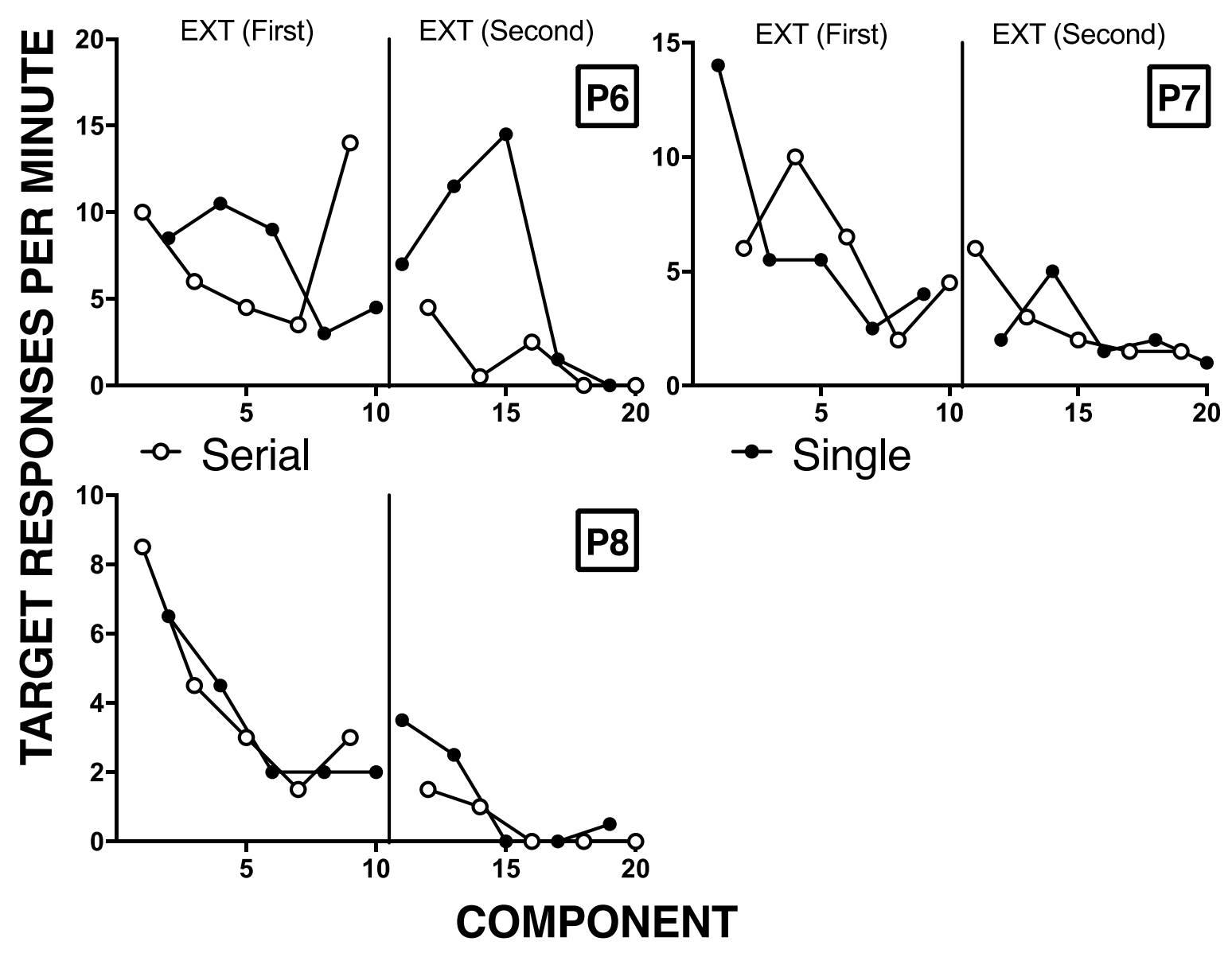

Figure 4. Results for participants who were interviewed. Each graph shows results for a participant. Filled circles denote target-response rates during the single-training component, and open circles denote target-response rates during the serial-training component. Y-axes values vary across participants. Condition labels show the first and second extinction phase. 


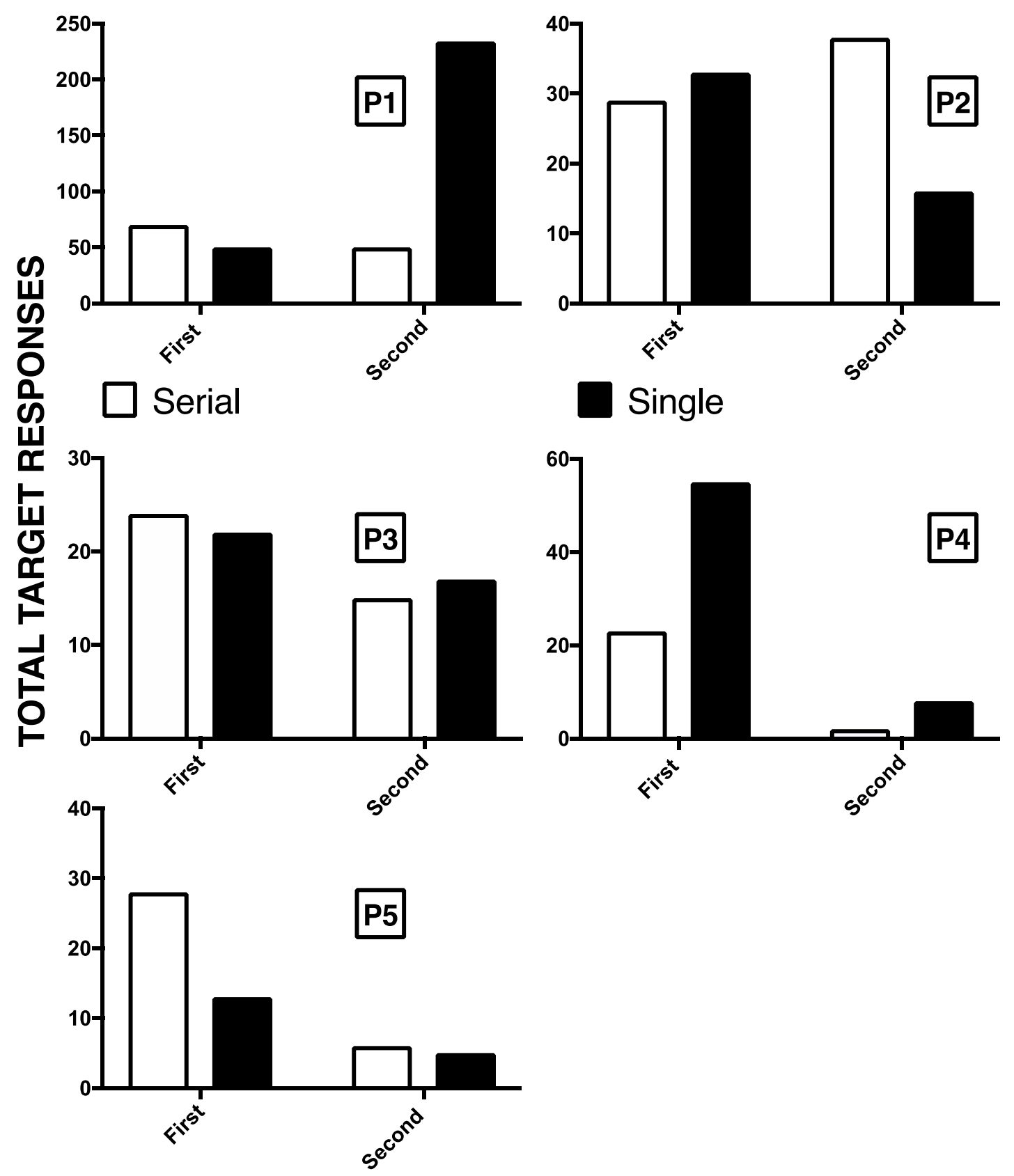

EXPOSURE

Figure 5. Results for participants who were not interviewed. Each graph shows results for a participant. Black bars denote total target responses during the single-training component, and white bars denote total target responses during the serial-training component. Y-axes values vary across participants. Total responses for the first exposure to extinction are on the left and total responses for the second exposure to extinction are on the right. 


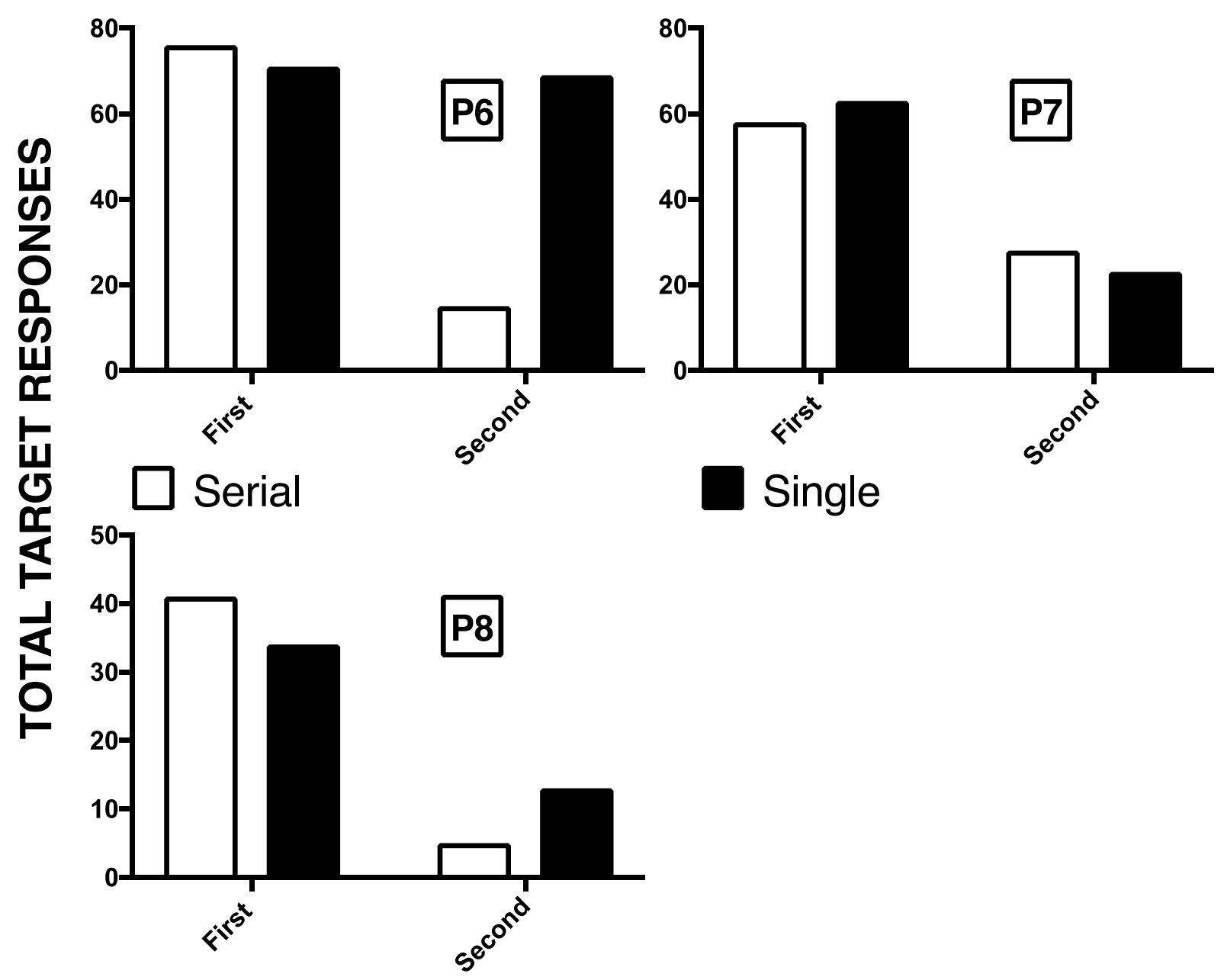

EXPOSURE

Figure 6. Results for participants who were interviewed. Each graph shows results for a participant. Black bars denote total target responses during the single-training component, and white bars denote total target responses during the serial-training component. Y-axes values vary across participants. Total responses for the first exposure to extinction are on the left and total responses for the second exposure to extinction are on the right. 


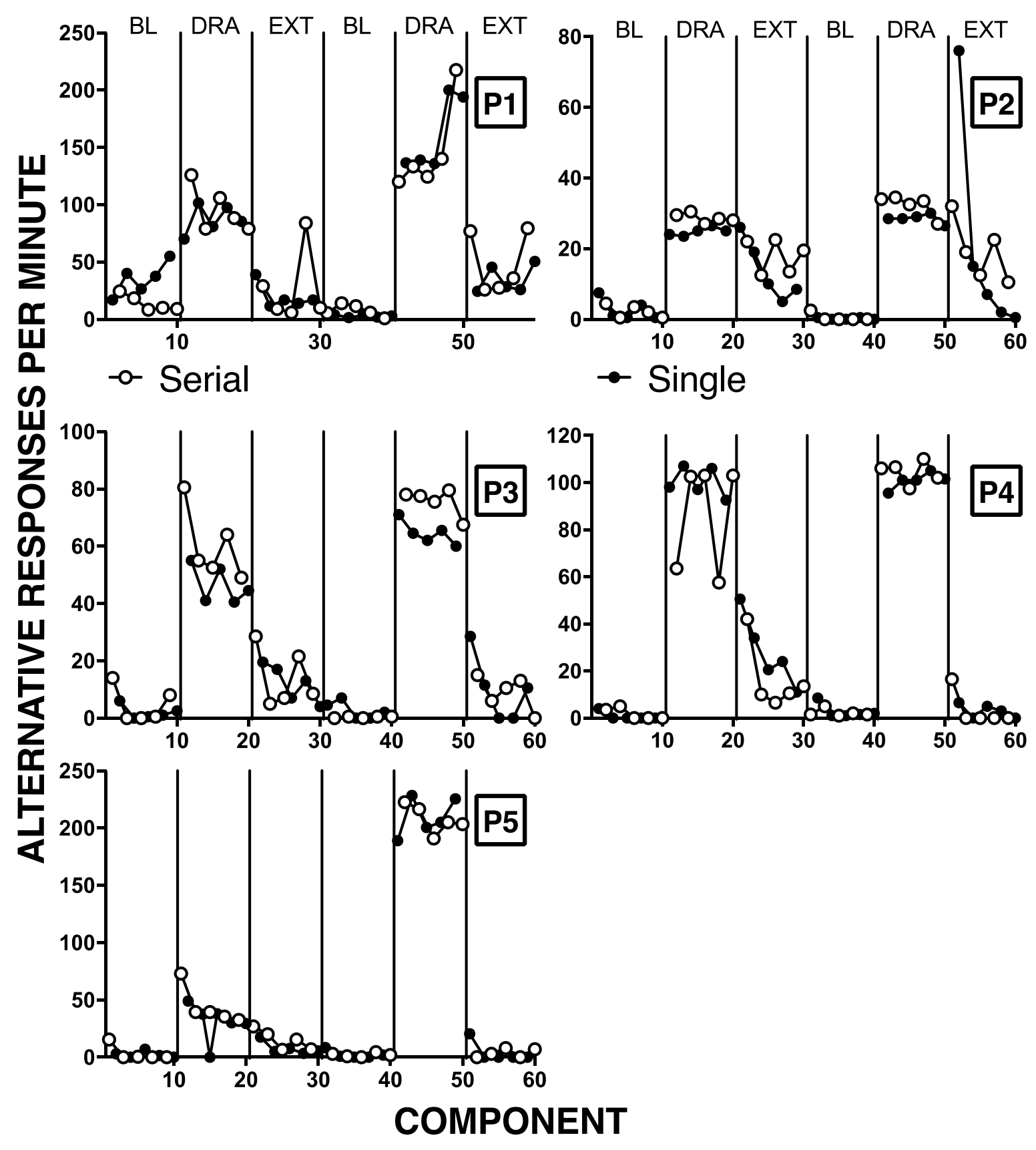

Figure 7. Results for participants who were not interviewed. Each graph shows results for a participant. Filled circles denote alternative-response rates during the single-training component, and open circles denote alternative response-rates during the serial-training component. Y-axes values vary across participants. Condition labels show baseline (BL), DRA, and extinction phases (EXT). 


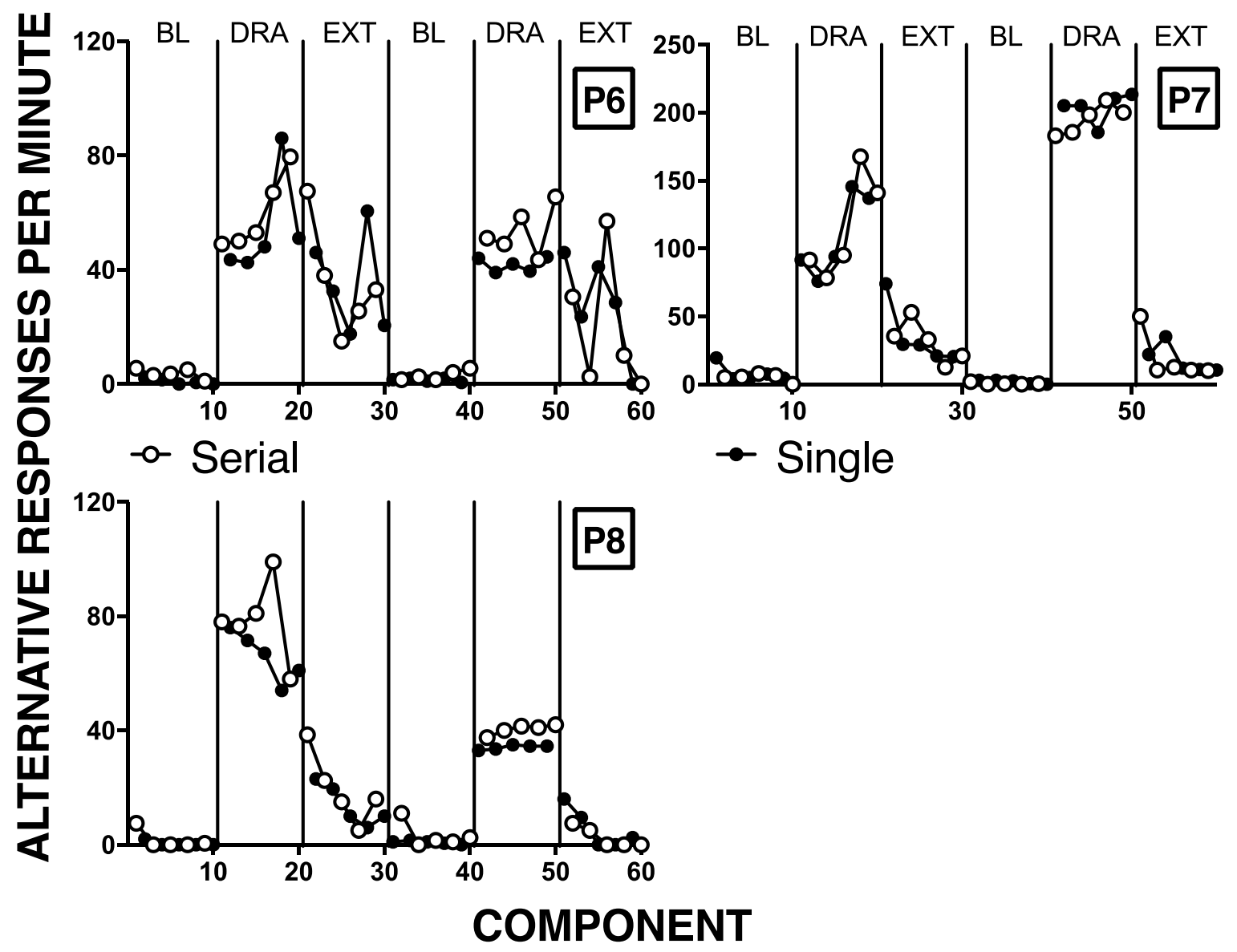

Figure 8. Results for participants who were interviewed. Each graph shows results for a participant. Filled circles denote alternative response-rates during the single-training component, and open circles denote alternative-response rates during the serial-training component. Y-axes values vary across participants. Condition labels show baseline (BL), DRA, and extinction phases (EXT). 